\title{
Continuum Topology Optimization of Buckling-Sensitive Structures
}

\author{
Salam Rahmatalla* and Colby C. Swan ${ }^{\dagger}$ \\ University of Iowa, Iowa City, Iowa 52242
}

\begin{abstract}
Two formulations for continuum topology optimization of structures taking buckling considerations into account are developed, implemented, and compared. In the first, the structure undergoing a specified loading is modeled as a hyperelastic continuum at finite deformations and is optimized to maximize the minimum critical buckling load. In the second, the structure under a similar loading is modeled as linear elastic, and the critical buckling load is computed with linearized buckling analysis. Specific issues addressed include usage of suitable "mixing rules," a node-based design variable formulation, techniques for eliminating regions devoid of structural material from the analysis problem, and consistent design sensitivity analysis. The performance of the formulations is demonstrated on the design of different structures. When problems are solved with moderate loads and generous material usage constraints, designs using compression and tension members are realized. Alternatively, when fairly large loads together with very stringent material usage constraints are imposed, structures utilizing primarily tension members result. Issues that arise when designing very light structures with stringent material usage constraints are discussed along with the importance of considering potential geometrical instabilities in the concept design of structural systems.
\end{abstract}

\section{Introduction and Motivation}

$\mathbf{V}$ ARIABLE topology material layout optimization is a potentially useful tool in the design of structures, mechanical parts, composite materials, and even microelectromechanical systems (MEMS). Its usage and a variety of formulations have been widely explored over the past 15 years for a considerable range of applications. ${ }^{1}$ Bendsoe and Kikuchi ${ }^{2}$ and numerous subsequent works by a wide range of investigators have introduced topology optimization methods dealing with linear elastic material behaviors and geometrically linear structural behavior. To this point comparatively few works have addressed continuum structural topology optimization of nonlinear systems. Among these are works using Voigt-Reuss continuum topology formulations and consistent sensitivity analysis techniques for designing structures ${ }^{3}$ and composite materials $\mathrm{s}^{4}$ featuring general materially nonlinear behaviors; maximizing structural ductility using adaptive topology optimization ${ }^{5}$; elastoplastic combined shape and topology optimization ${ }^{6}$; and continuum topology optimization of elastic systems undergoing finite deformations. ${ }^{7-12}$

Consideration of geometrical and/or buckling instabilities is an important issue in the conceptualdesign of sparse spatial structures. For example, in design of long-span bridges tension structures are typically optimal because they preclude potential buckling. If continuum topology optimization were applied to obtain concept designs of sparse spatial structures and buckling were not considered, the design results could rely excessively on compression and thus constitute unacceptable concept designs. In design optimization of discrete truss structures, buckling behaviors can be avoided altogether by prescription of Euler buckling constraints on loads in individual structural members. ${ }^{13,14}$ In continuum topology design formulations, however, this process is much more challenging because

Received 6 December 2001; revision received 9 October 2002; accepted for publication 1 February 2003. Copyright (C) 2003 by the American Institute of Aeronautics and Astronautics, Inc. All rights reserved. Copies of this paper may be made for personal or internal use, on condition that the copier pay the $\$ 10.00$ per-copy fee to the Copyright Clearance Center, Inc. 222 Rosewood Drive, Danvers, MA 01923; include the code 0001-1452/03 $\$ 10.00$ in correspondence with the CCC.

* Associate Professor, Department of Civil and Environmental Engineering, Center for Computer-Aided Design.

${ }^{\dagger}$ Ph.D. Candidate and Research Assistant, Department of Civil and Environmental Engineering, Center for Computer-Aided Design, 4120 Seamans Center for the Engineering Arts and Sciences; colby-swan@ uiowa.edu. Member AIAA. it is very difficult to identify discrete structural members, their geometrical properties, and their end support conditions from the vector of design variables. One promising approach to addressing geometrical instabilities in continuum structural topology optimization ${ }^{15}$ is to model the structure as a linearly elastic system and to use the minimum critical buckling load computed via eigenvalue analysis in either the objective function or as a design constraint.

On the other hand, in the design of MEMS with continuum structural topology optimization it is not uncommon for the system to be designed to undergo finite deformations even before geometric instabilities might develop. ${ }^{16,17}$ In such cases it is necessary to base the analysis and design on a more general framework that addresses finite deformations in the system and the development of potential geometrical instabilities. In recent years works dealing with continuum topology optimization of structures to minimize the generalized compliance at finite deformations have been proposed and demonstrated ${ }^{8}$, although minimization of nonlinearcompliance does not necessarily address potential geometrical instabilities. To this point in time, only a limited number of works ${ }^{9-12}$ have dealt with this important issue of taking finite deformations of the system into account, while also taking account of the associated instabilities that arise in the structure in a consistent fashion.

In the body of this paper, a general hyperelastic continuum framework is developed for structural analysis and continuum topology optimization. Within this framework both the generalized compliance of the system and the minimum critical buckling load for the system can be addressed. As an alternative, a linear elastic continuum topology formulation is also used along with minimum critical buckling values computed by linearized buckling analysis. For both formulations expressions for design sensitivity of structural responses to material distribution parameters are developed and verified. The two approaches have been implemented, tested, and compared on a variety of relevant design problems.

Although they are not the central focus of this work, two additional issues critical to achieving the objectives of this work are addressed in this manuscript. The first is that of using a continuum topology formulation based on nodal design variables as opposed to element-based design variables. Because node-based design variables feature $C^{0}$ continuity, this framework is invulnerable to checkerboarding instabilities and thus does not require any spatial filtering techniques to preclude such instabilities. As it is beyond the scope of this paper to fully explore the characteristics of nodal topology formulations, the subjecthas been exploredin considerable detail in a separate paper fully devoted to the issue. ${ }^{18}$ The second 
issue addressed is that of removing regions devoid of structural material from the structural analysis model. In the current context this is a vital consideration, because "empty" elements can suffer excessively large deformations as the structural system undergoes large deformations. The excessive deformation in the empty elements can lead to singular behaviors that make it extremely difficult to solve the nonlinear system equations. In addition, because the structural analysis problems considered here are frequently nonlinear the savings in computational effort when void regions are eliminated from the structural model can be very considerable.

\section{Distribution of Materials}

In most continuum structural optimization formulations there is some treatment of intermediate cases where a specific region of a structure is not fully occupied by solid structural material and yet not completely devoid of structural material either. In this work description of these regions uses amorphous mixtures (i.e., no specific microarrangement of materials is assumed). The complete undeformed spatial domain of the structure to be designed is denoted by $\Omega_{S}$; its designable subset by $\Omega_{D}$; and its nondesignable subset in which the spatial/topological arrangement of materials is taken to be fixed by $\Omega_{N}$. The arrangement of a structural material in $\Omega_{D}$ remains to be determined, and so this region is called designable. A set of single or multiple loading/boundary conditions to which $\Omega_{S}$ will be subjected is specified, and a starting design $\boldsymbol{b}^{(0)}$, which specifies the initial material layout in $\Omega_{D}$, is selected. For each set of loading/boundary conditions, the structure will be analyzed as a boundary-value problem.

The design of a structure is here considered to be the spatial distribution of the structural material in $\Omega_{s}$. To describe very general structural material distributions in $\Omega_{s}$, a volume-fraction approach described earlier in Ref. 3 and alternatively described as the density approach by others is used. Although preference is given here to final material layout distributions that are nearly discrete, such distributions are typically achieved using continuous formulations permitting mixtures to exist throughout the design domain $\Omega_{D}$. By permitting mixtures, the structural material $A$ and a fictitious void material $B$ are allowed to simultaneously and partially occupy an infinitesimal neighborhoodabout each Lagrangian point $X$ in $\Omega_{D}$. The volume fraction of structural material phase $A$ at a fixed Lagrangian point $\boldsymbol{X}$ in the design domain $\Omega_{D}$ is denoted by $\varphi_{A}(\boldsymbol{X})$ and represents the fraction of an infinitesimal volume element surrounding point $\boldsymbol{X}$ occupied by material $A$. Natural constraints upon the spatial volume fractions for the two-material problem are

$$
0 \leq \varphi_{A}(\boldsymbol{X}) \leq 1 \quad 0 \leq \varphi_{B}(\boldsymbol{X}) \leq 1 \quad \varphi_{A}(\boldsymbol{X})+\varphi_{B}(\boldsymbol{X})=1
$$

The last physical constraint of Eq. (1) states that the material volume fractions at $\boldsymbol{X}$ are not independentand so one need only be concerned with the layout of structural material $A$.

Using the same mesh and basis functions that will be used to solve the structural analysis problem described next, the spatial distribution of structural material in $\Omega_{S}$ is expressed using the following expansion:

$$
\varphi(\boldsymbol{x})=\sum_{i=1}^{\text {Numnp }} \varphi_{i} N_{i}(\boldsymbol{x}) \quad \forall \boldsymbol{x} \in \Omega_{S}
$$

where $\varphi_{i}$ are the nodal volume fractions, $N_{i}(\boldsymbol{x})$ are the nodal shape functions, and Numnp denotes the number of nodes in the structural model. The design vector $\boldsymbol{b}$ describing the arrangement of materials in the structural domain $\Omega_{S}$ thus has the composition $\boldsymbol{b}=\left(\varphi_{1}, \varphi_{2}, \varphi_{3}, \ldots, \varphi_{\text {Numn }}\right)$. This approach yields a $C^{0}$ continuous design variable field that is not susceptible to checkerboarding instabilities. ${ }^{18}$

\section{Analysis Formulation}

\section{A. Hyperelastic Structural Analysis at Finite Deformations}

The strong form of the nonlinear elliptic boundary-value problems to be solved is as follows: Find $\boldsymbol{u}:\left(\Omega_{S} \times[0, T]\right) \mapsto \mathfrak{R}^{3}$, such that the Kirchhoff stress field satisfies

$$
\tau_{i j, j}+\rho_{0} \gamma_{j}=0 \quad \text { on } \quad \Omega_{S} \quad \forall t \in[0, T]
$$

subject to the boundary conditions:

$$
\begin{array}{llll}
u_{j}(t)=g_{j}(t) \quad \text { on } \quad \Gamma_{g j} \quad \text { for } \quad j=1,2,3, & t \in[0, T] \\
n_{i} \tau_{i j}=h_{j}(t) \quad \text { on } \quad \Gamma_{h j} \quad \text { for } \quad j=1,2,3, & t \in[0, T]
\end{array}
$$

The Kirchhoff stress tensor $\tau$ is related to the Cauchy stress tensor $\sigma$ via the relation $\boldsymbol{\tau}=J \boldsymbol{\sigma}$ where $J=\operatorname{det}(\boldsymbol{F})$ and $\boldsymbol{F}$ is the deformation gradient tensor. As is customary, it is assumed that the Lagrangian surface $\Gamma$ bounding the Lagrangian structural domain $\Omega_{S}$ admits the decomposition $\boldsymbol{\Gamma}=\overline{\boldsymbol{\Gamma}_{g j} \cup \boldsymbol{\Gamma}_{h j}}$ and $\Gamma_{g_{j}} \cap \Gamma_{h_{j}}=\{\emptyset\}$ for $j=1,2,3$. For a given mesh discretization of $\Omega_{s}$ whose complete set of nodes is denoted $\{\eta\}$, the subsequent design formulation is facilitated by introducing a subset of nodes $\left\{\eta_{h}\right\}$ at which nonvanishing external forces are applied and a subset of nodes $\left\{\eta_{g}\right\}$ at which nonvanishing prescribed displacements are applied.

Because the analysis problem is being solved in the context of topologyoptimization, it is assumed that a local microscopic mixture of two generic materials $A$ and $B$ resides at each point $X$ in the structural domain $\Omega_{S}$. In Eq. (3a) $\tau$ represents the macroscopic Kirchhoff stress of the local mixture, which is dependent on the constitutive properties of the two material phases and the mixing rule employed,

$$
\boldsymbol{\tau}(\boldsymbol{X})=\boldsymbol{\tau}\left[\boldsymbol{\tau}^{A}\left(\boldsymbol{F}^{A}\right) ; \boldsymbol{\tau}^{B}\left(\boldsymbol{F}^{B}\right) ; \varphi\right]
$$

where $\boldsymbol{F}^{A}$ and $\boldsymbol{F}^{B}$ are the respective deformation gradients for materials $A$ and $B$ at a given point $X, \tau^{A}$ and $\tau^{B}$ are the stress tensors for the two materials at $\boldsymbol{X}$, and $\varphi$ is the volume fraction of material $A$. For simplicity and efficiency, a power law mixing rule with an isodeformation condition ${ }^{6}$ is used here. Therefore a specific form of Eq. (4) is

$$
\boldsymbol{\tau}(\boldsymbol{X})=\varphi^{p} \boldsymbol{\tau}^{A}+\left(1-\varphi^{p}\right) \boldsymbol{\tau}^{B}
$$

where $p \geq 1$ is a fixed parameter of the mixing rule. In accordance with the isodeformation assumption,

$$
\boldsymbol{F}^{A}=\boldsymbol{F}^{B}=\boldsymbol{F}=\frac{\partial \boldsymbol{x}}{\partial \boldsymbol{X}}
$$

where $\boldsymbol{x}=\boldsymbol{X}+\boldsymbol{u}(\boldsymbol{X})$.

It is assumed that both materials $A$ and $B$ obey isotropic hyperelastic constitutivelaws. The particularstrain energy function $E$ used here for both materials is that of Ciarlet ${ }^{19}$ wherein the volumetric $U$ and deviatoric $\bar{W}$ strain energy functions for the two materials $A$ and $B$ are assumed to be decoupled and of the forms:

$$
\begin{gathered}
E(\boldsymbol{F})=U(J)+\bar{W}(\boldsymbol{\theta}) \\
U(J)=\frac{1}{2} K\left[\frac{1}{2}\left(J^{2}-1\right)-\ln (J)\right] \\
\bar{W}=\frac{1}{2} \mu[\operatorname{tr}(\overline{\boldsymbol{\theta}})-3]
\end{gathered}
$$

In the preceding expression $J$ is again the determinant of $\boldsymbol{F} ; K$ is a constant bulk modulus; $\mu$ is a constant shear modulus; $\boldsymbol{\theta}=\boldsymbol{F} \boldsymbol{F}^{T}$ is the left Cauchy-Green deformation tensor; and $\overline{\boldsymbol{\theta}}=J^{-(2 / 3)} \boldsymbol{\theta}$ is its deviatoric part. For this model, therefore, the Kirchhoff stress $\tau$ in a material is thus related to deformation quantities in that material as follows:

$$
\boldsymbol{\tau}=J U^{\prime}(J) \mathbf{1}+2 \operatorname{dev} \frac{\partial \bar{W}}{\partial \boldsymbol{\theta}}
$$

The weak or variational form of the stress equilibrium problem can be obtained by restating the original form [Eq. (3a)] as

$$
\int_{\Omega_{S}}\left(\tau_{i j, i} \delta u_{j}+\rho_{0} \gamma_{j} \delta u_{j}\right) \mathrm{d} \Omega_{S}=0
$$


from which integration by parts, usage of Green's theorem, and utilization of the natural boundary conditions gives the virtual work equation

$$
\int_{\Omega_{S}} \tau_{i j} \delta \varepsilon_{i j} \mathrm{~d} \Omega_{S}=\int_{\Omega_{S}} \rho_{0} \gamma_{j} \delta u_{j} \mathrm{~d} \Omega_{S}+\int_{\Gamma_{h}} h_{j} \delta u_{j} \mathrm{~d} \Gamma_{h}
$$

In Eq. (10) the expression on the left represents the internal virtual work $\mathrm{d}\left(\delta W^{\text {int }}\right)$, and that on the right, the external virtual work $\mathrm{d}\left(\delta W^{\text {ext }}\right)$. The differential of the internal virtual work can be written as

$$
\mathrm{d}\left(\delta W^{\mathrm{int}}\right)=\int_{\Omega_{S}} \delta \varepsilon_{i j} \mathrm{~d} L_{v}\left(\tau_{i j}\right) \mathrm{d} \Omega_{S}+\int_{\Omega_{S}} \delta \varepsilon_{j i} \tau_{i m} \mathrm{~d} \varepsilon_{j m} \mathrm{~d} \Omega_{S}
$$

indicating a decomposition into, respectively, a material stiffness term containing the Lie differential of the Kirchhoff stress and a geometric stiffness term.

Usage of a Galerkin formulation, in which the real and variational kinematic fields are expanded in terms of the same nodal basis functions and discretization of the time domain into a finite number of discrete time points, leads to the following force balance equations at each unrestrainednode $A$ in the mesh as here at the $(n+1)$ th time step:

$$
\boldsymbol{r}_{n+1}^{A}=\left(\boldsymbol{f}^{\mathrm{int}}\right)_{n+1}^{A}-\left(\boldsymbol{f}^{\mathrm{ext}}\right)_{n+1}^{A}=0
$$

where

$$
\begin{gathered}
\left(\boldsymbol{f}^{\text {int }}\right)_{n+1}^{A}=\int_{\Omega_{S}}\left(\boldsymbol{B}^{A}\right)_{n+1}^{T}: \boldsymbol{\tau}_{n+1} \mathrm{~d} \Omega_{S} \\
\left(\boldsymbol{f}^{\text {ext }}\right)_{n+1}^{A}=\int_{\Omega_{S}} \rho_{0} N^{A} \gamma_{n+1} \mathrm{~d} \Omega_{S}+\int_{\Gamma_{h}} N^{A} \boldsymbol{h}_{n+1} \mathrm{~d} \Gamma_{h}
\end{gathered}
$$

In Eqs. (13a) and (13b), $\boldsymbol{B}_{n+1}^{A}$ represents the spatial infinitesimal nodal strain displacement matrix $\left[\boldsymbol{B}_{n+1}^{A}=\nabla_{x_{n+1}}^{s} N^{A}(\boldsymbol{x})\right]$, and $N^{A}$ denotes the nodal basis function for the $A$ th node. Under finite deformations Eq. (12) represents a set of nonlinear algebraic equations that must be solved in an iterative fashion for the incremental displacement field $(\Delta \boldsymbol{u})_{n+1}=\boldsymbol{u}_{n+1}-\boldsymbol{u}_{n}$ for each time step of the analysis problem.

When external forces applied to a structure are independent of its response, the derivative of the $i$ th residual force vector component at the $A$ th node with respect to the $j$ th displacement vector component of the $B$ th node is simply

$$
K_{i l}^{A B}=\int_{\Omega_{S}} B_{j i}^{A} c_{j k} B_{k l}^{B} \mathrm{~d} \Omega_{S}+\int_{\Omega_{S}} N_{, j}^{A} \tau_{j k} N_{, k}^{B} \delta_{i l} \mathrm{~d} \Omega_{S}
$$

where $c_{j k}$ is the spatial elasticity tensor in condensed form. Assembly of this nodal stiffness operator for all unrestrained nodes $A$ and $B$ gives the structural tangent stiffness matrix.

\section{B. Compliance Functional and Design Sensitivity Analysis}

The generalized compliance $\Pi$ of a structural system undergoing a loading by a system of varying body forces, applied tractions, and applied displacements can be expressed as follows:

$$
\begin{aligned}
\Pi(t) & =\int_{0}^{t} \int_{\Omega_{s}} \rho(\varphi) \gamma(\tau) \cdot \boldsymbol{v} \mathrm{d} \Omega_{s} \mathrm{~d} \tau \\
& +\int_{0}^{t} \int_{\Gamma_{h}} \boldsymbol{h}(\tau) \cdot \boldsymbol{v} \mathrm{d} \Gamma_{h} \mathrm{~d} \tau-\int_{0}^{t} \int_{\Gamma_{g}} \boldsymbol{t}^{n} \cdot \dot{\boldsymbol{g}}(\tau) \mathrm{d} \Gamma_{g} \mathrm{~d} \tau
\end{aligned}
$$

where $\gamma(\tau)$ is a time-varying body force vector, $\boldsymbol{h}(\tau)$ a system of surface tractions applied to the structure, and $\dot{\boldsymbol{g}}(\tau)$ a field of velocities applied to the structure, where in all cases $\tau$ plays the role of a parametric time variable. The generalized compliance of a structure can potentially be used to design structures in a way that minimizes their susceptibility to buckling. For example, when a structure goes through a point of instability the incremental compliance at that point gets very large. Consequently, it is possible that by minimizing the generalized compliance of a structural system one is also designing the structure to be less prone to buckling. For a wide variety of material behaviors, the generalized compliance of a structural system can be computed using integration as follows:

$$
\begin{aligned}
\Pi\left(t_{N}\right)= & \sum_{k \in\left\{\eta_{h}\right\}} \sum_{n=0}^{N-1}\left(f_{n+\frac{1}{2}}^{k}\right) \cdot\left(\Delta \boldsymbol{u}_{n+1}^{k}\right) \\
& \quad-\sum_{j \in\left\{\eta_{g}\right\}} \sum_{n=0}^{N-1}\left(f_{n+\frac{1}{2}}^{j}\right) \cdot\left(\Delta \boldsymbol{g}_{n+1}^{j}\right) \\
= & \sum_{n=0}^{N-1}\left[\sum_{k \in\left\{\eta_{h}\right\}}\left(f_{n+\frac{1}{2}}^{k}\right) \cdot\left(\Delta \boldsymbol{u}_{n+1}^{k}\right)-\sum_{j \in\left\{\eta_{g}\right\}}\left(f_{n+\frac{1}{2}}^{j}\right) \cdot\left(\Delta \boldsymbol{g}_{n+1}^{j}\right)\right] \\
= & \sum_{n=0}^{N-1}\left(\Delta \Pi_{n+1}\right)
\end{aligned}
$$

The first sum in Eqs. (16a) and (16b) corresponds to work done by external forces applied to the structure, where specific expressions for external forces were provided in Eq. (13b), and the second sum in Eqs. (16a) and (16b) expresses the work done on the structure by applied displacements. Displacement loading is applied to the structure with prescription of nonvanishing displacements $g$ at the nodal subset $\left\{\eta_{g}\right\}$. Such nodes need not necessarily lie on the external boundaries of $\Omega_{S}$, and the associated displacement field is simply

$$
\boldsymbol{g}(\boldsymbol{x})=\sum_{j \in\left\{\eta_{g}\right\}} N^{j}(\boldsymbol{X}) g_{j}
$$

where $g_{j}$ are applied nodal displacements.

The design derivative of the generalized structural compliance at any given time instant $t_{N}$ can be expressed using adjoint sensitivity analysis ${ }^{20}$ simply as

$$
\frac{\mathrm{d} \Pi\left(t_{N}\right)}{\mathrm{d} \boldsymbol{b}}=\sum_{n=0}^{N-1} \frac{\mathrm{d}\left(\Delta \Pi_{n+1}\right)}{\mathrm{d} \boldsymbol{b}}
$$

where

$$
\begin{aligned}
\frac{\mathrm{d}\left(\Delta \Pi_{n+1}\right)}{\mathrm{d} \boldsymbol{b}}=\sum_{k \in\left\{\eta_{h}\right\}}\left[\frac{\partial \boldsymbol{f}_{n+\frac{1}{2}}^{k}}{\partial \boldsymbol{b}} \cdot \Delta \boldsymbol{u}_{n+1}^{k}+\boldsymbol{f}_{n+\frac{1}{2}}^{k} \cdot \frac{\mathrm{d}\left(\Delta \boldsymbol{u}_{n+1}^{k}\right)}{\mathrm{d} \boldsymbol{b}}\right] \\
\quad-\sum_{j \in\left\{\eta_{g}\right\}} \frac{\mathrm{d} \boldsymbol{f}_{n+\frac{1}{2}}^{j}}{\mathrm{~d} \boldsymbol{b}} \cdot \Delta \boldsymbol{g}_{n+1}^{j} \\
=\sum_{k \in\left\{\eta_{h}\right\}}\left[\frac{\partial \boldsymbol{f}_{n+\frac{1}{2}}^{k}}{\partial \boldsymbol{b}} \cdot \Delta \boldsymbol{u}_{n+1}^{k}+\left(\boldsymbol{u}_{n+1}^{a}\right)^{k} \cdot \frac{\partial\left(\boldsymbol{r}_{n+1}\right)}{\partial \boldsymbol{b}}\right] \\
-\sum_{j \in\left\{\eta_{g}\right\}} \frac{\mathrm{d} \boldsymbol{f}_{n+\frac{1}{2}}^{j}}{\mathrm{~d} \boldsymbol{b}} \cdot \Delta \boldsymbol{g}_{n+1}^{j}
\end{aligned}
$$

In Eq. (18b), $\left(\boldsymbol{u}_{n+1}^{a}\right)^{k}$ is the solution of the following linear adjoint problem solved after the equilibrium analysis problem is solved at the $(n+1)$ th time/load step:

$$
\boldsymbol{K}_{n+1} \cdot\left(\boldsymbol{u}_{n+1}^{a}\right)^{k}=-\boldsymbol{f}_{n+\frac{1}{2}}^{k}
$$

In solving Eq. (19), the current tangent stiffness operator specified in Eq. (14) is employed.

\section{Critical Load and Design Sensitivity Analysis}

An alternative approach to designing buckling-resistantstructures is to apply displacement-controled loading to the structure and to 


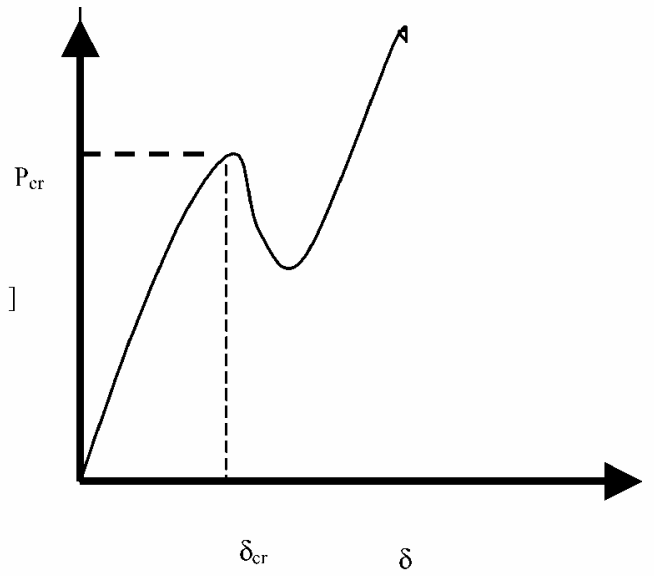

Fig. 1a Critical load and deflection associated with a structural response.

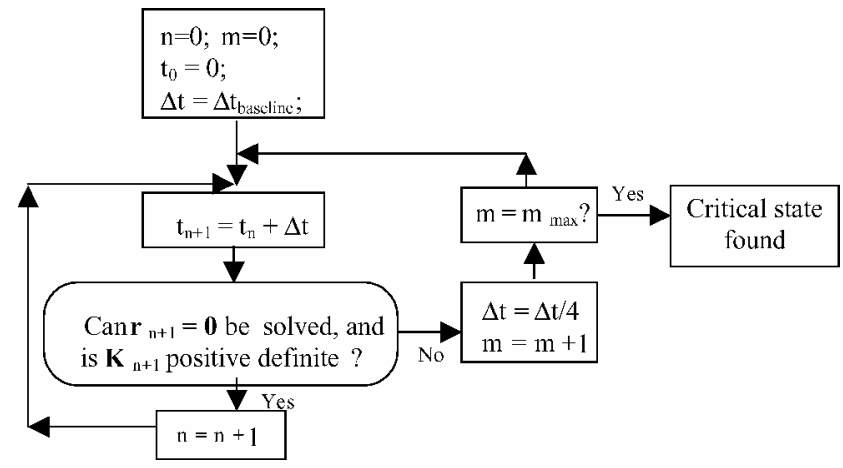

Fig. 1b Algorithm for finding the first critical point of a structure's response.

maximize the criticalinternal force that can be generated in response to this loading. The magnitude of the applied displacementloading is merely that which induces the first instabilityin the structural model. The first instability point is the first point in the load-deflection response of the structuralmodel at which the tangentstiffness operator defined in Eq. (14) becomes singular. A reliable algorithm for finding such singular points that has been used by the authorsin a variety of other applications $\mathrm{s}^{21,22}$ is presented in Fig. 1. Essentially, this algorithm involves gradual and iterative approaching of the structural model's first critical point of instability.

Once the critical point is found, the objective is to compute the magnitude of the resistance force generated in the structure and to design the structure so as to maximize the resistance. For example, if the applied displacement loading

$$
\boldsymbol{g}(\boldsymbol{x})=\sum_{K \in\left\{n_{g}\right\}} N^{K}(\boldsymbol{X}) \cdot g_{K}
$$

is applied to the structure and induces instability, then the magnitude of the critical resistance force generated in the structure will be

$$
f_{\text {crit }}^{\text {int }}=\sum_{K \in\left\{n_{g}\right\}}\left[\frac{-\boldsymbol{g}_{K}}{\left\|\boldsymbol{g}_{K}\right\|} \cdot \int_{\Omega} \boldsymbol{B}_{K}^{T} \cdot \boldsymbol{\tau} \mathrm{d} \Omega\right]
$$

Accordingly, an optimization problemto maximize this critical force would be as follows:

$$
\max _{b}\left(f_{\text {crit }}^{\text {int }}\right) \quad \text { such that } \boldsymbol{r}(\boldsymbol{b}, \boldsymbol{u})=0
$$

$$
\text { and such that } \quad V_{\text {Material }}-V_{\text {allowable }} \leq 0
$$

The design derivative of the critical buckling force is computed using adjoint sensitivity analysis as follows:

$$
\frac{\mathrm{d} f_{\text {crit }}^{\text {int }}}{\mathrm{d} \boldsymbol{b}}=\sum_{K \in\left\{n_{g}\right\}}\left[\frac{-\boldsymbol{g}_{K}}{\left\|\boldsymbol{g}_{K}\right\|} \cdot \int_{\Omega} \boldsymbol{B}_{K}^{T} \cdot \frac{\partial \boldsymbol{\tau}}{\partial \boldsymbol{b}} \mathrm{d} \Omega+\left(\boldsymbol{u}_{K}^{a}\right) \cdot \frac{\partial \boldsymbol{r}}{\partial \boldsymbol{b}}\right]
$$

where $\left(\boldsymbol{u}_{K}^{a}\right)$ is the adjoint displacementassociated with the $K$ th node at which prescribed displacements are applied. Specifically, it is the solution of the following adjoint problem:

$$
\boldsymbol{K} \cdot\left(\boldsymbol{u}_{K}^{a}\right)=-\frac{\partial\left(f_{\text {crit }}^{\mathrm{int}}\right)_{K}}{\partial \boldsymbol{u}}
$$

where $\boldsymbol{K}$ is tangent stiffnessoperator at the current state of the model.

\section{Linearized Buckling Model}

\section{A. Analysis}

The full geometrically nonlinear method just proposed for maximizing the minimum critical buckling load in continuum structural topology optimization is potentially very computationally expensive. A potentially more efficient alternative might be to use linearized buckling eigenvalue analysis to estimate the critical buckling load for the structure and to then maximize this approximation Neves et al. ${ }^{15}$ have presented an approach to include the critical load criterion in the continuum topology optimization model. Linearized buckling eigenvalue analysis proceeds as follows: A prescribed force loading $f^{\text {ext }}$ is applied to the structure with its magnitude necessarily being less than that required to induce geometric instability in the structure. Once the resulting linear, elastostatic displacement solution $\boldsymbol{u}=\left\{u_{i}\right\} \in \boldsymbol{R}^{N}$ to the applied loading $\boldsymbol{f}^{\text {ext }}$ is obtained $\left(\boldsymbol{K}_{L} \cdot \boldsymbol{u}=\boldsymbol{f}^{\text {ext }}\right)$, where $\boldsymbol{K}_{L}$ is the linearized stiffness matrix, then the following eigenvalue problem is solved:

$$
\boldsymbol{K}_{L}(\boldsymbol{b}) \boldsymbol{\psi}+\lambda \boldsymbol{G}(\boldsymbol{u}, \boldsymbol{b}) \psi=0
$$

In the preceding, $\boldsymbol{b}=\left\{\boldsymbol{b}_{e}\right\} \in \boldsymbol{R}^{M}$ is the vector of design variables; $\boldsymbol{K}_{L}$ is the linear tangent stiffness operator; $\boldsymbol{G}(\boldsymbol{u}, \boldsymbol{b})$ is the linearized geometric stiffness matrix; $\lambda=-\boldsymbol{\psi}^{T} \boldsymbol{K}_{L} \boldsymbol{\psi} / \psi^{T} G \psi$ is an eigenvalue denoting the magnitude by which $f^{\text {ext }}$ must be scaled to create instability in the structure, and $\psi$ is a normalized eigenvector satisfying $\boldsymbol{\psi}^{T} \boldsymbol{K}_{L} \boldsymbol{\psi}=1$. For this model it is assumed that linearized stiffness operator $\boldsymbol{K}_{L}$ is real, symmetric, and positive definite, whereas $\boldsymbol{G}$ is only assumed to be real and symmetric. To avoid numerical difficulties in the solution of Eq. (24) associated with the indefinite characteristics of $\boldsymbol{G}$, it is common ${ }^{23}$ to solve the modified eigenvalue problem that deals with two positive definite matrices:

$$
\left[\left(\boldsymbol{K}_{L}+\boldsymbol{G}\right)-\gamma \boldsymbol{K}_{L}\right] \psi=0
$$

where

$$
[\gamma=(\lambda-1) / \lambda]
$$

\section{B. Optimization Problem}

Once the linearized eigenvalue problem [Eq. (25)] is posed and solved, the design problem is formulated to maximize the minimum buckling load $\lambda$. The objective functional $f_{E}$ to be minimized for this problem would simply be the reciprocal of the lowest eigenvalue $\lambda$ as follows:

$$
f_{E}(\boldsymbol{u}, \boldsymbol{b})=1 / \min (\lambda)
$$

The optimization problem is thus stated to minimize the reciprocal of the first (or minimum) critical buckling load as follows:

$$
\min _{b, u} f_{E}=\min _{b, u}\left(\frac{1}{\lambda}\right)=\min _{b, u}\left(-\max _{\psi \neq 0} \frac{\boldsymbol{\psi}^{T} \boldsymbol{G} \boldsymbol{\psi}}{\boldsymbol{\psi}^{T} \boldsymbol{K}_{L} \boldsymbol{\psi}}\right)
$$

subject to the normal bound constraints on the design variables [Eq. (1)], the linear, structural equilibrium state equation $\left[\boldsymbol{r}(\boldsymbol{u}, \boldsymbol{b})=0=\boldsymbol{K}_{L} \cdot \boldsymbol{u}-\boldsymbol{f}^{\mathrm{ext}}\right]$, and a constraint on material resources.

Using adjoint design sensitivity analysis, the Lagrangian of the optimization problem (27) is

$$
L=f_{E}+\boldsymbol{u}^{a} \cdot \boldsymbol{r}(\boldsymbol{u}, \boldsymbol{b})
$$


where $\boldsymbol{u}^{a}$ is the adjoint displacement vector and the solution of the linear, adjoint problem

$$
\boldsymbol{K}_{L} \boldsymbol{u}^{a}=\boldsymbol{\psi}^{T} \frac{\partial \boldsymbol{G}}{\partial \boldsymbol{u}} \boldsymbol{\psi}
$$

For a simple eigenvalue the design gradient of $L$ is written simply as

$$
\frac{\mathrm{d} L}{\mathrm{~d} \boldsymbol{b}}=\frac{\partial f_{E}}{\partial \boldsymbol{b}}+\left(\boldsymbol{u}^{a}\right)^{T} \cdot \frac{\partial \boldsymbol{r}}{\partial \boldsymbol{b}}
$$

where:

$$
\frac{\partial f_{E}}{\partial \boldsymbol{b}}=-\boldsymbol{\psi}^{T}\left(\frac{\partial \boldsymbol{G}}{\partial \boldsymbol{b}}+\frac{1}{\lambda} \frac{\partial \boldsymbol{K}_{L}}{\partial \boldsymbol{b}}\right) \boldsymbol{\psi}
$$

In Eq. (30) the partial derivative of $\boldsymbol{r}$ with respect to $\boldsymbol{b}$ can be found from the equilibrium state equation as follows:

$$
\frac{\partial \boldsymbol{r}}{\partial \boldsymbol{b}}=\frac{\partial \boldsymbol{K}_{L}}{\partial \boldsymbol{b}} \cdot \boldsymbol{u}-\frac{\partial \boldsymbol{f}^{\mathrm{ext}}}{\partial \boldsymbol{b}}
$$

Consequently, the final expression for the design gradient of $L$ can be written in the following form:

$$
\frac{\mathrm{d} L}{\mathrm{~d} \boldsymbol{b}}=-\boldsymbol{\psi}^{T}\left(\frac{\partial \boldsymbol{G}}{\partial \boldsymbol{b}}+\frac{1}{\lambda} \frac{\partial \boldsymbol{K}_{L}}{\partial \boldsymbol{b}}\right) \boldsymbol{\psi}+\left(\boldsymbol{u}^{a}\right)^{T}\left(\frac{\partial \boldsymbol{K}_{L}}{\partial \boldsymbol{b}} \cdot \boldsymbol{u}-\frac{\partial \boldsymbol{f}^{\mathrm{ext}}}{\partial \boldsymbol{b}}\right)
$$

In the current formulation a simple eigenvalue has been assumed. In cases where multiple eigenvaluesoccur, computation of the sensitivities can be somewhat more involved because the eigenvectors of the repeated eigenvalues are not unique, rendering the eigenvalues only directionally differentiable.$^{24,25}$ Frequently, though certainly not always, however, multiple eigenvalues occur in symmetrical structures and are actually attributable to the structural symmetry. ${ }^{26}$ In such cases approaches that reduce the design space in accordance with the symmetry ${ }^{27}$ can render the repeated eigenvalues fully differentiable in the reduced design space.

\section{Analysis Problem Size Reduction Technique}

In continuum topology optimization of sparse structures with limited structural material usage and realistically large design loads, geometrical instabilities become a definite possibility. If modeled, geometrical instabilities in structural systems can result in finite deformations. The modeling of finite deformations in mixed solid-void grid-likemeshes used in continuumstructuraltopologyoptimization can result in excessive distortion of void or low-density elements that can in turn lead to numerical difficulties solving the structural analysis problem. Because the optimization process in continuum topology optimization typically removes structural material from low stress or low-sensitivity areas, fairly substantial regions of lowdensity elements are common. As these elements are highly compliant, they contribute very little to structural stability, while being subject to excessive deformation that creates numerical difficulties. Therefore, it is sometimes advantageous to identify these large regions of void and low-density elements and to remove them, at least temporarily, from the structural analysis problem. An automated algorithm for identifying such regions and removing them from the structural analysis problem is presented and discussed next. The procedure proposed and investigated here is reversible in that it permits low-density regions of the structure to return as high-density structural regions even after they have previously been removed from consideration during structural analysis.

The essence of the proposed analysis problemreductiontechnique can be captured in the three steps listed here:

1) All finite elements in the structural analysis model that are devoid of solid material, or nearly so, are identified as "void" elements. (Typically, in the examples presented next, if an element's volume fraction of solid material is less than or equal to 0.002 , it is identified as void.)
2) All nodes that are members only of void elements are identified as "prime" nodes. The degrees of freedom of such prime nodes are restrained, reducing the size of the analysis problem.

3) If only prime nodes comprise an element, that element is then denoted as a prime element. Such prime elements are then neglected in the structural analysis problem, so that if they undergo excessive distortion it does not create any singularities in the system of finite element equations. It is worth noting that prime elements are those that are surrounded by void elements.

A graphicaldescription supporting the explanation of this technique for reducing the analysis problem is presented in Fig. 2.

The matter of reducing the analysis problem by neglecting significant regions of void elements has been addressed in preceding works. ${ }^{9,10}$ The current reduction techniques have proven to be both robust and efficient in all of the example problems presented in Section VI. The techniques are especially powerful and effective when applied in design problems involving extremely sparse structures because highly refined meshes are needed when very stringent material resource constraints are imposed. When a fine mesh is employed with a very limited amount of structural material, the proposed reduction techniques will allow for dramatic savings in computing effort.

\section{Demonstrative Examples}

\section{A. Material Properties and Mixing Rules}

Before any sample computations were performed, the design gradient expressions presented in Secs. III and IV of this paper were confirmed on a number of small problems by comparison with converged finite difference gradients.In all cases nearly exact agreement was obtained between the results computed with the semianalytical expressions and the results computed with finite difference. With the design gradient expressions confirmed, a number of sample problems were then solved. In all example problems solved next, the initial starting designs always utilized a completely solid structural domain. In addition, the solid structural material in all problems was isotropic with Young's modulus of $307 \mathrm{GPa}$ and shear modulus of $118 \mathrm{GPa}$. The nodal design variable formulation of Sec. II was employed without any spatial filtering of design variables and without any perimeter control. Furthermore, the powerlaw mixing rule with $p=4$ was used in all computations. It is recognized that while using $(p=4)$ will generally give essentially discrete final design solutions it will not necessarily lead to attainment of the globally optimal design solution. Although continuation methods ${ }^{28}$ could have been employed here in which one begins the design problem with $(p=1)$ and then gradually increments $p$ until $(p=4)$, these are not guaranteed to converge to the globally optimal solution either.

\section{B. X-Structure Problem}

In this problem an X-shaped structure shown in Fig. 3a is considered. The lateral by vertical dimensions of the frame are $(11 \times 40)$. A displacement loading of $d \leq 10$ is applied to the center node of this structure, and for all designs the algorithm of Fig. 1 is applied together with geometrically nonlinear structural analysis to compute the critical internal buckling force that develops in response to the applied displacement loading. The design problem solved is that posed in Eq. (21), where only half of the original structural volume can be employed in the final, optimized structure. The structure is meshed with 1200 bilinear continuum finite elements, and the optimization problem is solved using sequential linear programming techniques. The design solution to this particular version of the problem is shown in Fig. 3b. Because it employs strictly tension as opposed to compression, it is indeed the correct solution that one would expect.

Although optimization of structures to maximize the minimum critical buckling load based on fully nonlinear analysis was shown to be successful, the computational expense can be considerable. As a potentially more efficient way to achieve the same objective, linearized buckling analysis can also be used. Thus, for the same structural model shown in Fig. 3a a force of magnitude $\left\|f^{\text {ext }}\right\|=1.9 \times 10^{12}$ was applied to the center node, and the structural 


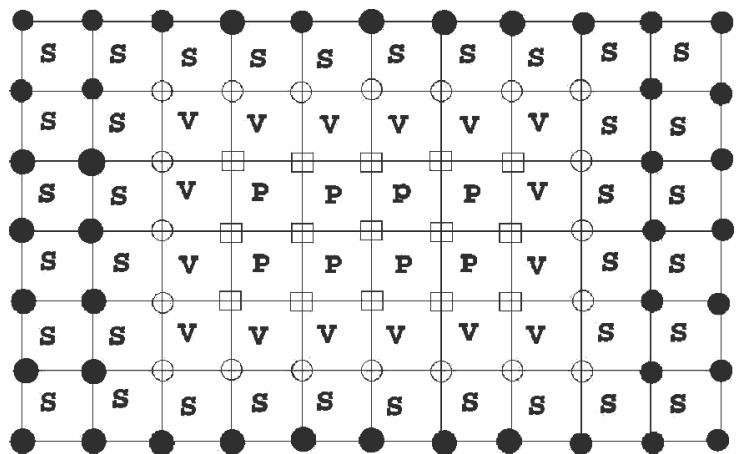

a)

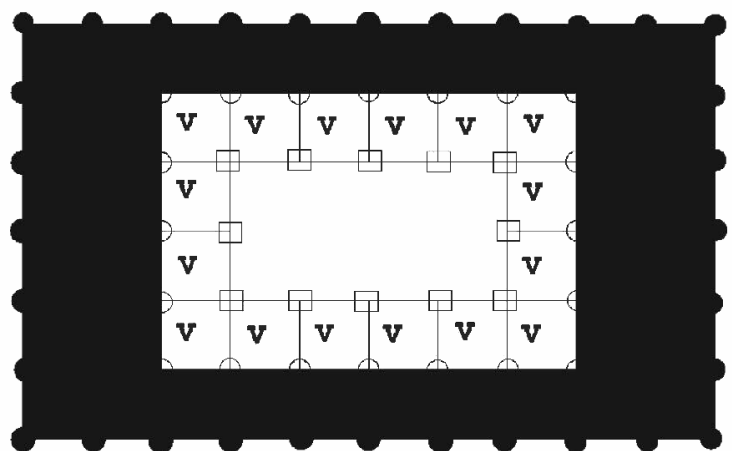

b)

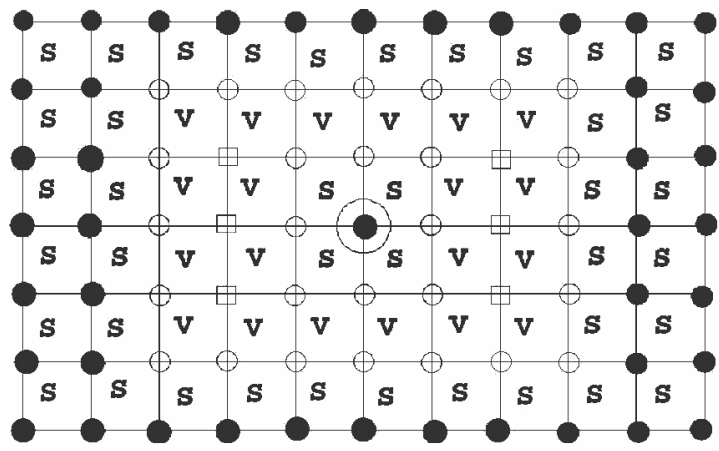

c)

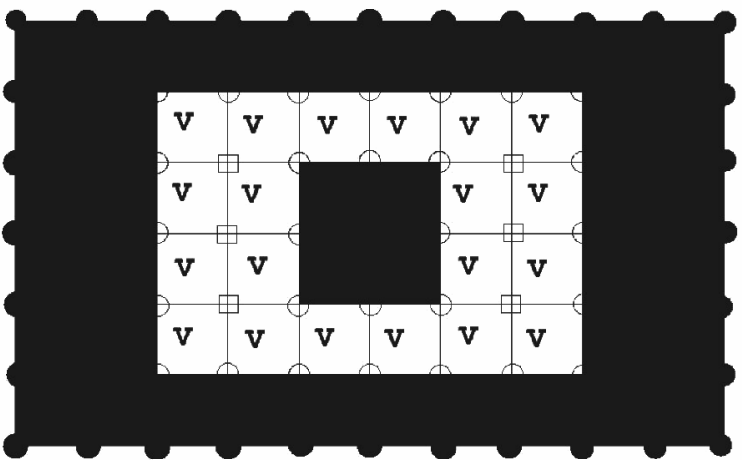

d)

Fig. 2 Schematic to illustrate analysis problem reduction technique: $\bigcirc$, nodes with associated design variable values of zero; $\bullet$, nodes with nonzero design variable values; $\square$, "prime" nodes whose degrees of freedom are restrained; elements with $S$ are at least partially solid; elements with $V$ are essentially devoid of solid material; elements with $P$ are prime elements removed from consideration, a) mesh with designated nodes and elements, b) corresponding mesh used in analysis with solid elements blackened, prime elements removed, and void elements retained, c) mesh with an isolated solid node among a region of void nodes, and d) resulting mesh in which there are no prime elements removed, thus avoiding formation of an island that is disconnected from the remainder of the mesh.

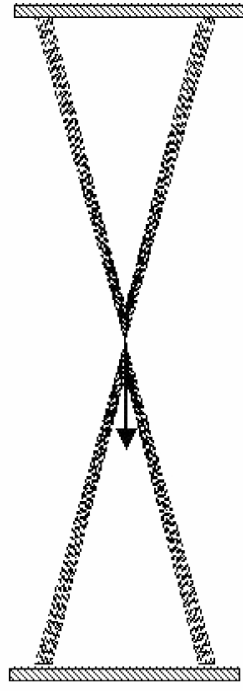

a)

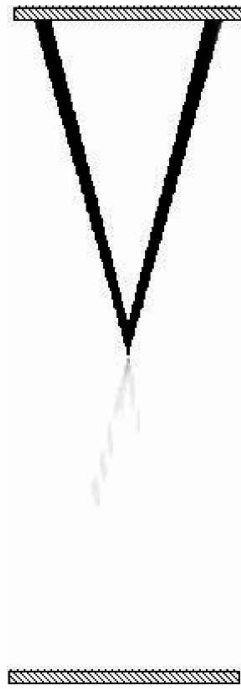

b)
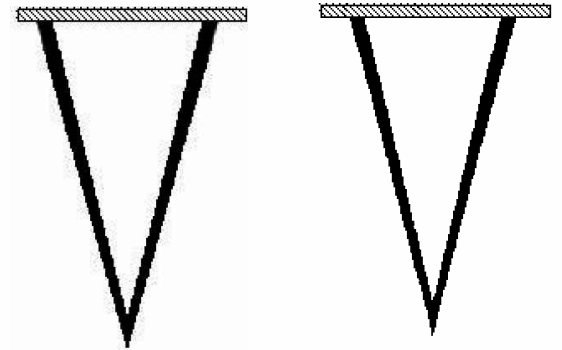

c) d)

Fig. 3 X-frame problem: a) structure dimensions $11 \times 40$ and modeled with a mesh of 1200 bilinear continuum elements is loaded as shown; b) design solution obtained by maximizing the minimum critical buckling load; c) design solution obtained by maximizing the minimum critical load obtained using linearized buckling analysis; and d) design solution obtained by minimizing the structural compliance up to the point of the first instability.

optimization problem was solved once again as follows:

$\min _{b}\left(\frac{1}{\lambda}\right)$ such that $\boldsymbol{r}(\boldsymbol{b}, \boldsymbol{u})=0$ for an applied load $\boldsymbol{f}^{\text {ext }}$

and such that $\quad V-V_{\text {allowable }} \leq 0$

With this linearized buckling criteria the resulting design (Fig. 3c) is virtually identical to that achieved with nonlinear analysis and shown in Fig. 3b. However, the computational time required with the linearized buckling analysis was significantly smaller than that required with the geometrically nonlinear analysis.

The preceding design problem was solved yet again to minimize the generalized compliance of the structure $(16 a-16 c)$ under the applied displacement loading up to the first point of instability. In this particular instance the resulting design obtained to minimize generalized compliance and shown in Fig. $3 \mathrm{~d}$ is comparable to the 


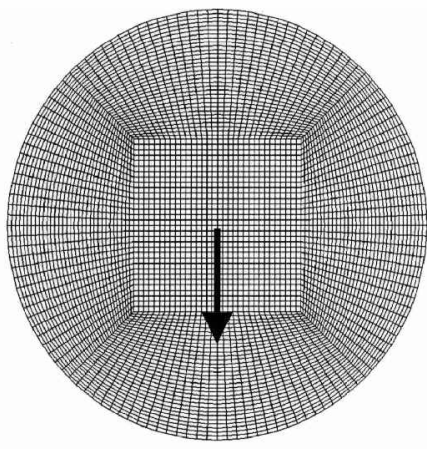

a)

d)

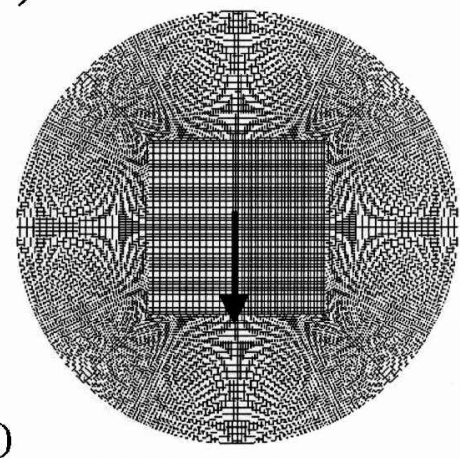

b)

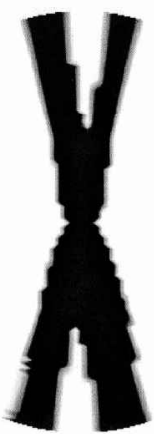

e)

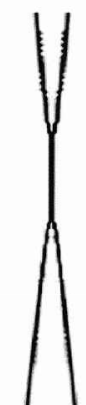

c)
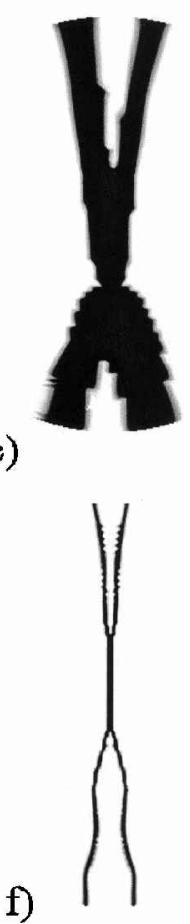

Fig. 4 Circle problem 1: a) circular structural domain modeled with coarse mesh, with rigid boundary conditions and point load or displacement applied at center; b) undeformed design solution obtained by minimizing structural compliance up to first critical point with structural material constraint at $20 \%$ of structural domain; c) deformed configuration of design solution; d) fine mesh and applied load; e) undeformed design solution obtained by minimizing structural compliance up to first critical point with structural material constraint at $2.5 \%$ of structural domain; and f) deformed configuration of design solution.

preceding designs obtained with nonlinear and linearized buckling analysis.

\section{Circle Problem}

Continuum topology optimization solutions are frequently used only as starting concepts that are suggestive of potentially optimal structural forms. That is, the designs produced are then taken to a second stage where more detailed shape and sizing analysis is performed. When used in design of large-scale structures, continuum topology solutions are frequently quite "heavy" in that the ratio of volume occupied by structural material to the enclosed structural volume can be unrealistically large. Specifically, the global volume fraction constraint used in many continuum topology optimization methods is typically in the range of $0.10-0.50$, whereas in many structures the structural material occupies only approximately $1-2 \%$ of the enclosed structural volume. Thus, although buckling of compression members can be a real concern in design of sparse structures, continuum topology methods that produce unrealistically heavy designs are unable to address such concerns in the concept design stage.

To address the development of geometric instabilities associated with buckling, continuum topology optimum methods must be used with a finite deformation nonlinear formulation or with a linearized buckling approach. In addition, sufficiently stringent material usage constraints must be imposed, which can in turn require highly refined structural meshes. To illustrate this point, we consider the design of a structure to carry a radial point load applied at the center of the structural domain to the fixed boundary of the circular domain of Fig. 4a. The analysis problem was solved by imposing a finite displacement $\delta \leq 0.1 R$ at the center node of the structure and using a nonlinear formulation to compute the resulting structural response. The design optimization problem was first solved with a coarse mesh (Fig. 4a) to minimize the generalized compliance of the structure up to the first point of instability. A structural material global volume fraction constraint of $20 \%$ was imposed. The resulting solution shown in Fig. 4b (undeformed) and Fig. 4c (deformed) is quite heavy and supports the applied load using both tension and compression. If during secondary design, however, the members are made much lighter, the possibility of buckling in the compression members would then become apparent, and the design would need to be changed significantly.

To detect the onset of geometrical instability within continuum structural topology optimization, it is sometimes necessary to solve problems of this type with much smaller volumes of structural material or with loads and/or displacements large enough to generate instability. The problem was solved once again to minimize the generalized compliance with the finer mesh shown in Fig. $4 \mathrm{~d}$ and a structural material constraint of $2.5 \%$ of the total structural volume. The resulting design is shown in Fig. 4e (undeformed) and Fig. 4f (deformed). Clearly, the structural model detects instability in the light compression members, yet the problem formulation does not result in complete elimination of the compression member.

As an alternative to solving this "circle" problem using a generalized compliance objective function, it was solved again using the minimum critical buckling loads as the objective function. Using the moderately fine mesh shown in Fig. 5a, the design problem was solved a number of times. It was first solved with material usage constraints of $25 \%$ of the structural volume and with applied an applied displacement $\delta \leq 0.1 R$, resulting in the design shown in Fig. $5 \mathrm{~b}$ (undeformed) and $5 \mathrm{c}$ (deformed). Because the material usage constraintis generous and because the loading is not necessarily large enough to generate structural instability, a design using both compression and tension is produced. When the design displacement loading is increased to $\delta \leq 0.8 R$ and when the material usage constraint is reduced to $5 \%$ and even to $2.5 \%$ of the total structural volume, the purely tensile designs of Figs. $5 \mathrm{~d}$ and $5 \mathrm{f}$ (undeformed) and $5 \mathrm{e}$ and $5 \mathrm{~g}$ (deformed) are obtained. Such designs are clearly preferred because they will not be vulnerable to buckling instabilities A similar solution (Figs. 5h and 5i) can be obtained by maximizing the minimum critical buckling load obtained using linearized buckling analysis.

In the preceding design problems relatively fine meshes were required in order to achieve sparse structural designs. The computational cost associated with using fine mesh can be high. However, when the problem reduction techniques of Sec. V are employed the cost can be reduced considerably. At the start of the design process, 


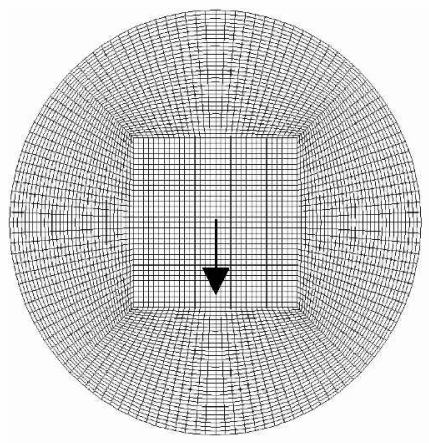

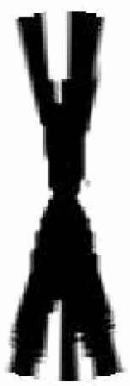

(b)

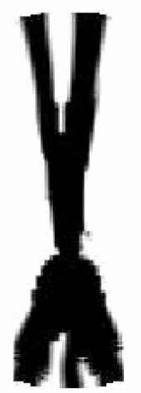

(c)

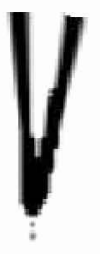

(d)

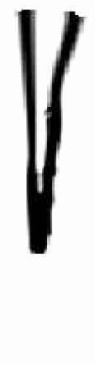

(e)
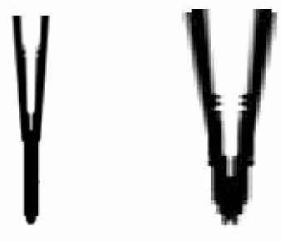

(f)

(h)
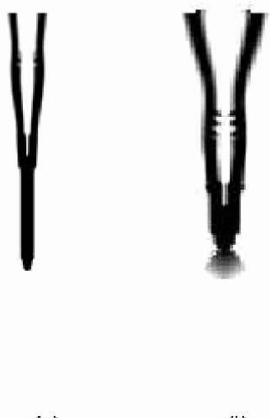

(1)

Fig. 5 Circle problem 2: a) circular structural domain with rigid boundary restraints and central load; b) undeformed solution obtained to maximize minimum critical nonlinear load with material constraint at $20 \%$ of structural volume and applied displacement loading $\delta \leq 0.1 R$; c) deformed configuration of associated design; d) undeformed design obtained to maximize minimum critical nonlinear load with material constraint at $5 \%$ of structural volume and applied displacement loading $\delta \leq 0.8 R$; e) deformed configuration of associated design; f) undeformed design obtained to maximize minimum critical nonlinear load with material constraint at $2.5 \%$ of structural volume and applied displacement loading $\delta \leq 0.8 R$ using finer mesh; g) deformed configuration of associated design; h) design obtained to maximize minimum critical load obtained with linearized buckling analysis; and i) deformation associated with critical mode.

the structural analysis problems are indeed quite large and expensive. However, as material is eliminated from the structural model as the optimization process continues, the analysis problem gets progressively smaller, until at the optimal design, the actual analysis problem can be quite modest in size. For example, in treating the circle problem the analysis computation time at the optimal designs was reduced by more than a factor of 10 using the proposed technique.

\section{Fixed-End Beam Problem}

In this problem a fixed-end beam is loaded vertically with a large displacement applied to the center of the upper edge of the beam as shown in Fig. 6a. The vertical and horizontal dimensions of the beam are $20 \times 40$, and the structural material usage constraint for this problem is $10 \%$ of the total structural volume. A similar problem was introduced and solved by Buhl et al., ${ }^{9}$ who showed that their formulation yields completely different designs when using geometrically linear analysis and when using geometrically nonlinear analysis. More recently Gea and Luo ${ }^{11}$ have studied a similar problem with three concentrated loads applied to the upper edge of the beam, obtaining design results similar to those presented in Ref. 9. In the present work both buckling formulations introduced earlier are applied to this problem in concert with the size reduction technique mentioned in Sec. V. In the nonlinear problem formulation a large displacement of 15 is applied to the structure, while a force $f=1.9 \times 10^{6}$ is applied in the linearized buckling case. The continuum topology optimized material layout designs obtained are shown in Figs. $6 \mathrm{~b}$ and 6e. The resulting topologies signify the ability of both methods to track and address the geometrical instabilities occurring in the structure, by building long tension members and comparatively short compression members. The design produced by linearized buckling analysis is noticeably shallower than that produced by the nonlinear analysis, and, as a result, uses shorter and thicker compression members. These concept design results are also in general agreement with those of Refs. 9 and 11.
To compare the relative performance of the design solutions shown in Fig. 6, the design (Fig. 6b) obtained with the nonlinear analysis formulation has a computed nonlinear buckling load magnitude of $2.4 \times 10^{8}$, whereas the design (Fig. 6e) computed from the linear analysis has a computed nonlinear buckling load magnitude of $7.51 \times 10^{7}$. Furthermore, when both designs are subjected to linearized buckling analysis the design from nonlinear analysis (Fig. 6b) has a linearized buckling factor of $\lambda=1 \times 10^{3}$, whereas that computed with linear analysis (Fig. 6e) has $\lambda=1.96 \times 10^{3}$. The authors do not take the differences between these two designs too seriously, however, because the designs shown are viewed as "concept designs," which are starting points for subsequent shape and sizing design optimization procedures.

In Ref. 9 the authors concluded that usage of a hyperelastic constitutive law such as that used by Bruns and Tortorelli ${ }^{8}$ appeared to result in unstable nonlinear analysis problems. In all of the preceding example problems, the hyperelastic constitutive model of Sec. III has been used with very good results in the nonlinear formulation together with the analysis problem size reduction technique of Sec. V.

\section{Discussion}

In this paper the objective has been to develop continuum structural topology optimization formulations that can be used to detect and avoid buckling instabilities in the conceptual design stage of sparse structural systems. Toward this end, both a finite deformation hyperelastic treatment of the structure and linear elastic, linearized buckling treatment of the structure have been developed and implemented. With the hyperelastic structural treatment both generalized compliance and the minimum critical buckling load were separately considered as objective functions. Based on the example problems solved, selection of the minimum critical buckling load as the objective function appears to be more effective at consistently achieving stable designs than the generalized compliance of the structural system. The minimum critical buckling load 


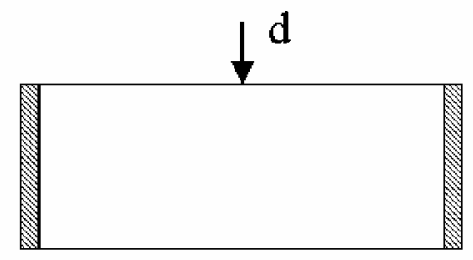

(a)

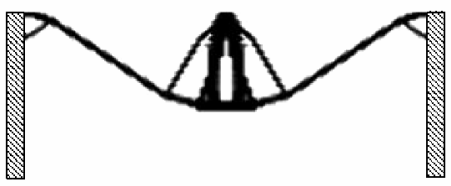

(b)

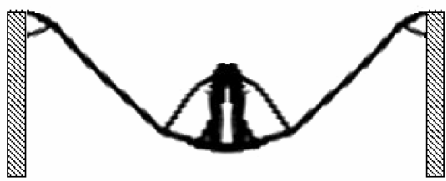

(c)

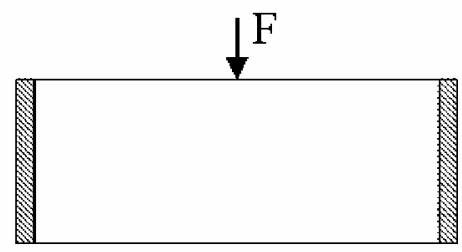

(d)

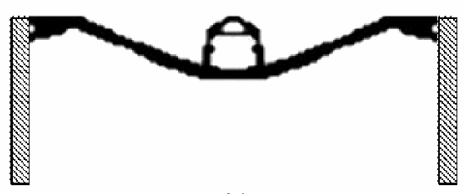

(e)

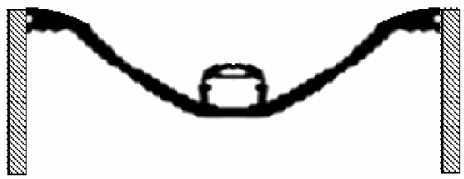

(f)

Fig. 6 Fixed-end beam problem: a) design problem with applied displacement loading for which structure is to be designed to maximize the minimum critical load computed via nonlinear analysis; b) associated design solution; and c) deformed shape of design solution; d) design problem with applied force loading for which structure is to be optimized to maximize minimum critical load computed with linearized buckling analysis; e) associated design solution; and f) deformed shape associated with buckling eigenmode.

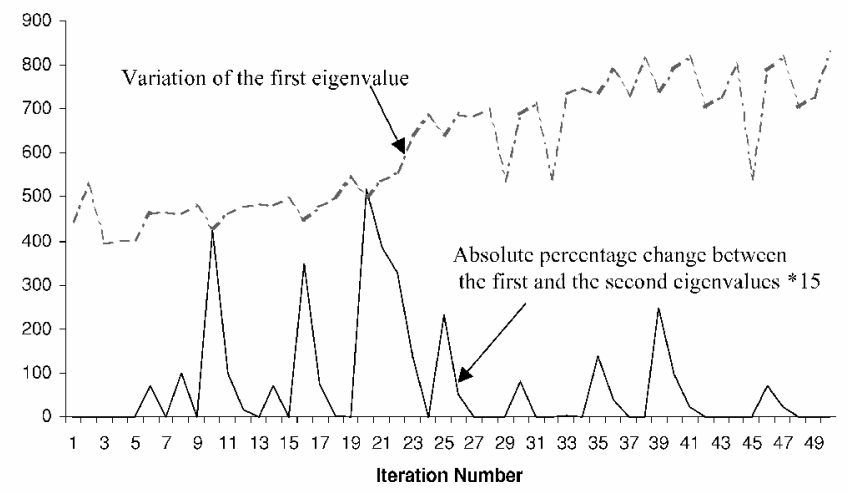

Fig. 7 Upper graph shows the variation in the first buckling eigenvalue during the optimization process, and the lower graph depicts the normalized difference between the first two eigenvalues during the optimization process.

as computed from linearized buckling eigenvalue analysis was also considered as an objective function in this work and was found to give design results roughly comparable to those produced from nonlinear stability analysis. For example, the performance of the linearized buckling solution shown in Fig. 6e was analyzed using the nonlinear buckling analysis and the nonlinear solution shown in Fig. 6b was analyzed by linearized buckling formulation. For this problem the raw performance characteristics of the design produced by the linearized buckling formulation (Fig. 6e) are comparable to that of the design produced by the nonlinear buckling formulation.

Because the buckling design optimization formulation here produced designs of raw performance characteristics comparable to that of the nonlinear formulation and because the structural analysis required in each design iteration is only linear, it is tempting to say that the linearized buckling formulation is clearly superior to the nonlinear buckling formulation. It must be noted, however, that repeated, nonsimple eigenvalues in the linearized formulation pose a potentially problematic issue. In the fixed-end beam example of Sec. VI.D, nonsimple repeated eigenvaluesnot attributable to structural symmetry occurred frequently in the design iterations (Fig. 7). When this occurred, the computed gradient information supplied from the sensitivity expression of Eq. (33) was incorrect, and so the optimization process typically made little or no progress toward an optimal solution for an iteration or two. When very small move limits $(0.01)$ were employed in the sequential linear programming optimization method, however, the design process quickly recovered and continued a broad (though not monotonic) trend of convergence toward an optimal design. With this approach the issue of nondifferentiability (or directional differentiability) of repeated eigenvalues was successfully overcome. Nevertheless, this is an issue that requires further investigation.

A structural analysis problem size reduction technique has been successfully implemented and tested on the numerous linear and nonlinear problems presented in this work. This technique reduces the problem size considerably and at the same time removes the unstable elements temporarily from the structural domain. In addition, the nodal design variable approach utilized in this work has demonstrated stable performance on all test problems with no propensity toward checkerboarding designs.

\section{Conclusions}

Within the context of a nodal topology optimization framework, alternative formulations have been implemented and tested for designing sparse structures with high buckling stability factors. Usage of generalized compliance as an objective function was found unsuitable for reliably yielding structural designs of high stability. Alternatively, two design formulations that maximized the buckling stability factors, one computed with full geometric nonlinearity and the other computed with linearized stability analysis, were found to reliably yield designs of high buckling stability. The formulation based on linearized stability analysis is much more computationally efficient than the formulation based on nonlinear analysis. Nevertheless, differentiability issues associated with nonsimple repeated buckling eigenvalues require further attention.

\section{Acknowledgments}

This research was funded in part by the University of Iowa CIFRE Program and in part by National Science Foundation Grant DMS9874015. Richard K. Miller (Olin College) is also acknowledged for helpful discussions and recommending the circle problem as a good test for the proposed design methods. 


\section{References}

${ }^{1}$ Eschenauer, H. A., and Olhoff, N., "Topology Optimization of Continuum Structures: A Review," Applied Mechanics Reviews, Vol. 54, No. 4, 2001, pp. 331-389.

${ }^{2}$ Bendsoe, M. P., and Kikuchi, N., "Generating Optimal Topology in Structural Design Using a Homogenization Method," Computer Methods in Applied Mechanics and Engineering, Vol. 71, 1988, pp. 197-224.

${ }^{3}$ Swan, C. C., and Kosaka, I., "Voigt-Reuss Topology Optimization for Structures with Nonlinear Material Behaviours," International Journal for Numerical Methods in Engineering, Vol. 40, 1997, pp. 3785-3814.

${ }^{4}$ Swan, C. C., and Arora, J. S., "Topology Optimization of Material Layout in Structured Composites of High Stiffness and High Strength," Structural Optimization, Vol. 13, No. 1, 1997, pp. 45-59.

${ }^{5}$ Maute, K., Schwarz, S., and Ramm, E., "Adaptive Topology Optimization of Elastoplastic Structures," Structural Optimization, Vol. 15, 1998, pp. 81-91.

${ }^{6}$ Schwarz, S., Maute, K., and Ramm, E., "Topology and Shape Optimization for Elastoplastic Structural Response," Computer Methods in Applied Mechanics and Engineering, Vol. 190, 2001, pp. 2135-2155.

${ }^{7} \mathrm{Jog}$, C., "Distributed-Parameter Optimization and Topology Design for Non-Linear Thermoelasticity," Computer Methods in Applied Mechanics and Engineering, Vol. 132, 1996, pp. 117-134.

${ }^{8}$ Bruns, T. E., and Tortorelli, D. A., "Topology Optimization of NonLinear Elastic Structures and Compliant Mechanisms," Computer Methods in Applied Mechanics and Engineering, Vol. 190, 2001, pp. 3443-3459.

${ }^{9}$ Buhl, T., Pedersen, W., and Sigmund, O., "Stiffness Design of Geometrically Nonlinear Structures Using Topology Optimization," Journal of Structural and Multidisciplinary Optimization, Vol. 19, 2000, pp. 93-104.

${ }^{10}$ Pedersen, C. B. W., Buhl, T., and Sigmund, O., "Topology Synthesis of Large-Displacement Compliant Mechanisms," International Journal for Numerical Methods in Engineering, Vol. 50, 2001, pp. 2683-2705.

${ }^{11}$ Gea, H. C., and Luo, J., "Topology Optimization of Structures with Geometrical Nonlinearities," Computers and Structures, Vol. 79, 2001,pp. $1977-$ 1985.

${ }^{12}$ Saxena, A., and Ananthasuresh, G. K., “Topology Synthesis of Compliant Mechanisms for Nonlinear Force-Deflection and Curved Path Specifications," Journal of Mechanical Design, Vol. 123, 2001, pp. 33-42.

${ }^{13}$ Rozvany, G. I. N., "Difficulties in Truss Topology Optimization with Stress, Local Buckling and System Stability Constraints," Structural Optimization, Vol. 11, 1996, pp. 213-217.

${ }^{14}$ Achtziger, W., "Local Stability of Trusses in the Context of Topology Optimization Part II: A Numerical Approach,” Structural Optimization, Vol. 17, 1999, pp. 247-258.

${ }^{15}$ Neves, M. M., Rodrigues, H., and Guedes, J. M., “General Topology
Design of Structures with a Buckling Load Criterion," Structural Optimization, Vol. 10, 1995, pp. 71-78.

${ }^{16}$ Sigmund, O., "On the Design of Compliant Mechanisms Using Topology Optimization,” Mechanics of Structures and Machines, Vol. 25, No. 4, 1997, pp. 495-526.

${ }^{17}$ Larsen, U., Sigmund, O., and Bouwstra, S., "Design and Fabrication of Compliant Micro-Mechanisms and Structures with Negative Poisson's ratio," Journal of Microelectromechanical Systems, Vol. 6, 1997, pp. 99106.

${ }^{18}$ Rahmatalla, S. F., and Swan, C. C., "A Q4/Q4 Continuum Structural Topology Optimization Formulation," Journal of Structural and Multidiciplinary Optimization (to be published).

${ }^{19}$ Ciarlet, P. G., Mathematical Elasticity, Volume I: Three-Dimensional Elasticity, Elsevier, Amsterdam, 1988.

${ }^{20}$ Cardoso, and Arora, J. S., "Adjoint Sensitivity Analysis for Nonlinear Dynamic Thermoelastic Systems,” AIAA Journal, Vol. 29, No. 2, 1991, pp. 253-263.

${ }^{21}$ Swan, C. C., and Seo, Y.-K., "Limit State Analysis of Earthen Slopes Using Dual Continuum/FEM Approaches," International Journal for Numerical and Analytical Methods in Geomechanics, Vol. 23, 1999, pp. 13591371.

${ }^{22}$ Swan, C. C., and Seo, Y.-K., "Stability Analysis of Embankments on Saturated Soils Using Elasto-Plastic Porous Medium Models," Journal of Geotechnical and Geoenvironmental Engineering, Vol. 127, No. 5, 2001 pp. 436-445.

${ }^{23}$ Bathe, K. J., Finite Element Procedures, Prentice-Hall, Upper Saddle River, NJ, 1996, pp. 630-636.

${ }^{24}$ Seyranian, A. P., Lund, E., and Olhoff, N., "Multiple Eigenvalues in Structural Optimization Problems," Structural Optimization, Vol. 8, 1994 pp. 207-227.

${ }^{25}$ Ohsaki, M., and Uetani, K., "Sensitivity Analysis of Bifurcation Load of Finite-Dimensional Symmetric Systems," International Journal for Numerical Methods in Engineering, Vol. 39, 1996, pp. 1707-1720.

${ }^{26}$ Pedersen, N. L., "Maximization of Eigenvalues Using Topology Optimization," Structural Optimization, Vol. 20, 2000, pp. 2-12.

${ }^{27}$ Kosaka, I., and Swan, C. C., "A Symmetry Reduction Method for Continuum Structural Topology Optimization," Computers and Structures, Vol. 70, 1999, pp. 47-61.

${ }^{28}$ Swan, C. C., and Kosaka, I., "Voigt-Reuss Topology Optimization for Structures with Linear Elastic Material Behaviors," International Jour nal for Numerical Methods in Engineering, Vol. 40, 1997, pp. 3033 3057 .

E. R. Johnson Associate Editor 